ARTICLE

DOI: $10.1038 / s 41467-017-01504-5$

\title{
Virtual photons in the ground state of a dissipative system
}

\author{
Simone De Liberato (1) ${ }^{1}$
}

Much of the novel physics predicted to be observable in the ultrastrong light-matter coupling regime rests on the hybridisation between states with different numbers of excitations, leading to a population of virtual photons in the system's ground state. In this article, exploiting an exact diagonalisation approach, we derive both analytical and numerical results for the population of virtual photons in presence of arbitrary losses. Specialising our results to the case of Lorentzian resonances we then show that the virtual photon population is only quantitatively affected by losses, even when those become the dominant energy scale. Our results demonstrate most of the ultrastrong-coupling phenomenology can be observed in loss-dominated systems which are not even in the standard strong coupling regime. We thus open the possibility to investigate ultrastrong-coupling physics to platforms that were previously considered unsuitable due to their large losses.

\footnotetext{
${ }^{1}$ School of Physics and Astronomy, University of Southampton, Southampton SO17 1BJ, UK. Correspondence and requests for materials should be addressed to S.D.L. (email: S.De-Liberato@soton.ac.uk)
} 
$\mathrm{T}$ he study of the interaction between light and matter has been one of the cornerstones in the development of quantum mechanics. In most cases the light-matter coupling is weak enough to be intuitively described in terms of the emission and absorption of photons, while the matter system jumps between two of its quantised eigenstates. When the resonant coupling of an optically active transition with a mode of the electromagnetic field is larger than the losses determining their respective linewidths, it becomes possible to spectroscopically resolve the splitting due to the interaction. The system is then said to be in the strong light-matter coupling regime. Contrary to the weak coupling case, here the interaction between light and matter cannot be described in terms of emission and absorption of photons, but it is necessary to consider the dressed light-matter excitations of the coupled system. Finally, if the coupling becomes even larger, comparable with the bare frequencies of the excitations, we enter a third regime, called ultrastrong coupling. Such a regime, described ${ }^{1}$ and achieved ${ }^{2}$ for the first time using intersubband polaritons, has since been studied both theoretically and experimentally in a variety of different systems $s^{3-17}$. Interest in this novel regime has been fuelled by its rich phenomenology, including quantum phase transitions ${ }^{18-20}$, modification of energy transport $^{21,22}$ and optical properties ${ }^{23-27}$, and the possibility to use it to influence chemical and thermodynamic processes ${ }^{28-32}$.

The relevant dimensionless parameter in a perturbative treatment of the light-matter interaction is the ratio between the coupling and the bare excitation frequencies. In the ultrastrongcoupling regime such a parameter becomes non-negligible, with values larger than one recently achieved ${ }^{33}$. Higher-order perturbative effects due to the antiresonant terms in the Hamiltonian, which do not conserve the number of excitations, are then able to hybridise states with different numbers of excitations. Such an hybridisation is at the origin of much of the ultrastrong-coupling phenomenology ${ }^{25-27}$ and one of its most striking consequences is that the ground state $|G\rangle$ becomes a squeezed vacuum state containing a finite population of virtual photons. Those photons are said to be virtual because the ground state cannot radiate. Their presence can be however directly revealed when the system parameters are non-adiabatically modulated in time, transmuting virtual photons into real ones ${ }^{34-45}$, a process which presents strong analogies with the dynamical Casimir effect ${ }^{46-50}$ and with the Hawking radiation ${ }^{51-53}$. Non-adiabatic modulation of the parameters of a light-matter coupled system has been experimentally achieved in dielectric systems by modifying the dipole density with a femtosecond laser puls ${ }^{54}$, and in superconducting circuits by applying an external flux bias ${ }^{55,56}$. Another promising proposal in this direction is the use of the superconducting to classical transition to alter the resonator response ${ }^{57}$.

While the best way to correctly model losses in the ultrastrongcoupling regime has been object of much attention ${ }^{58-60}$, their impact on the structure of the ground state and on the presence of virtual photons has been for the moment almost totally neglected. One of the reasons is that interest in ultrastrong coupling has historically emerged from the study of strongly coupled systems. Its achievement is usually demonstrated by fitting the resonant splitting of the coupled resonances to measure the coupling strength. Any system in which ultrastrong coupling has been demonstrated was thus a fortiori also in the strong coupling regime. But in this situation the loss rate is the smallest frequency scale of the problem, and perturbative methods that neglect its impact on the structure of the ground state are totally justified. Nevertheless strong and ultrastrong coupling depend on different figures of merit, and they are thus a priori independent regimes. Systems in the ultrastrong but not in the strong coupling regime could still have large couplings, as well as large losses, both comparable with the bare frequency of the optical transition. The ground state of the system would then also hybridise with its environment, modifying its structure. A thorough investigation of the effect of the losses on the virtual photon population in those systems then becomes necessary to ascertain whether ultrastrongcoupling phenomenology can still be observed or if it is completely quenched by the effect of the environment.

In this article we calculate through a non-perturbative procedure the virtual photon population in presence of arbitrary losses. Specialising our results to the case of Lorentzian light and matter resonances we prove that losses do have an impact on the virtual photon population, but only a quantitative one. Even in presence of dominant losses a sizeable fraction of virtual photons remains. Ultrastrong-coupling phenomena can thus be observed in systems with very large couplings, in which losses have impeached the observation of strong coupling for intrinsic, or technological reasons. Prime examples could be graphene single and bilayers in which, notwithstanding different theoretical calculations predicting very large dipoles ${ }^{16,17}$, strong coupling has not yet been achieved. Another example are hybrid quantum systems which were recently highlighted as ideal platforms for some quantum vacuum emission scheme $e^{34}$. These have only very recently ${ }^{61}$ achieved strong coupling, as they are characterised by large los$\operatorname{ses}^{62,63}$.

\section{Results}

Analytical expression for the virtual photon population. The quantity of interest for us will be the photonic population in the ground state $|G\rangle$ of the coupled light, matter and environment fields. In our treatment this quantity is also the measure of the hybridisation between states with different numbers of excitations. Due to the regime we are interested in, with all the parameters a priori of the same order, a perturbative approach would be unreliable and we are thus obliged to perform a nonperturbative calculation. In a light-matter coupled system energy can be lost through different channels. Photons can escape out of the system, or they can be absorbed by the matter excitation and their energy non-radiatively dissipated. The general theory we developed, detailed in the Supplementary Note 4, shows that in the considered parameter range the ground state photonic population essentially depends on the total amount of losses, regardless of their origin. Without loss of generality in the main body of the paper we will thus consider the case of losses due to absorption in a dielectric medium, which allows to obtain analytically intuitive results in terms of the complex dielectric function. In this case, considering an homogeneous dissipative dielectric with complex dielectric function $\epsilon(\omega)$, we show in the Methods section that the number of ground-state virtual photons in the mode $\mathbf{k}$ is given by

$$
N_{k}=\sum_{j} \operatorname{Im}\left[\frac{\Omega_{j}^{2}-c^{2} k^{2}}{4 \pi c^{3} k^{2}} \frac{\mathrm{d} \Omega_{j}}{\mathrm{~d} k}\left(i \pi-2 \log \left(\Omega_{j}\right)\right)\right]-\frac{1}{2},
$$

where the $\Omega_{j}$ are the solution of the dispersion equation

$$
\epsilon(\omega) \omega^{2}-c^{2} k^{2}=0
$$

located in the first quadrant of the complex plane. The quantity $N_{k}$ also represents the number of photons with wavevector $\mathbf{k}$ emitted upon an instantaneous switch-off of the interaction ${ }^{1}$ : after the switch-off the ground state would be the standard, empty vacuum and all the virtual photons would be free to radiate. Notice that this identification remains valid in presence of losses because without light-matter coupling there can be no absorption and all the virtual photons in the ground state are emitted. 
a

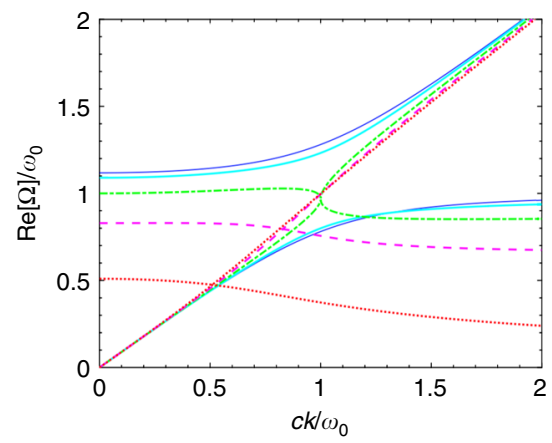

b

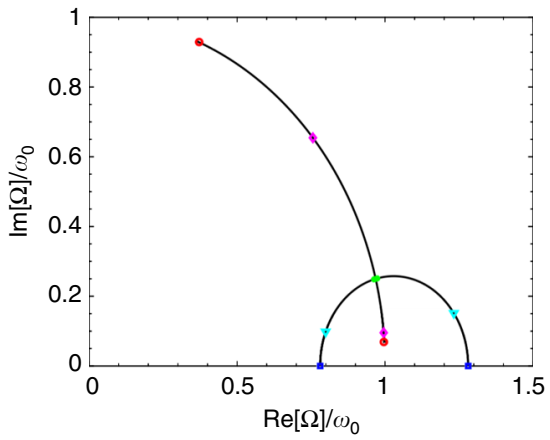

Fig. 1 Eigenmodes of the Lorentz model. a Dispersion of the two polaritonic branches from the Lorentz model in Eq. (3), for $\omega_{\mathrm{C}}=0.5 \omega_{\mathrm{O}}$ and $\gamma_{\mathrm{L}}=0$ (thin blue lines), $0.5 \omega_{0}$ (solid cyan lines), $\omega_{0}$ (dash-dotted green lines), $1.5 \omega_{0}$ (dashed magenta lines) and $2 \omega_{0}$ (dotted red lines). The transition between the strong coupling regime presenting an anticrossing (blue and cyan lines) and the weak one in which the polaritonic modes cross (magenta and red lines), with the green line at the edge between the two, is cleary visible. $\mathbf{b}$ Trajectories drawn by the eigenfrequencies in the complex plane, at resonance $c k=\omega_{0}$, when varying $\gamma_{\llcorner}$. Coloured squares, triangles, dots, diamonds and circles mark the increasing values of $\gamma_{\llcorner}$used in a

a

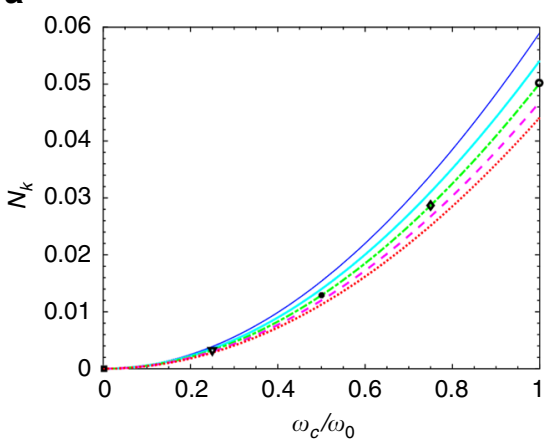

b

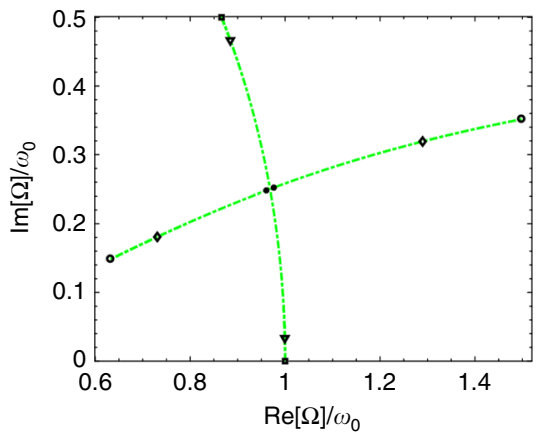

Fig. 2 Virtual photons in the resonant Lorentz model. a Number of photons in the resonant mode $c k=\omega_{0}$ as a function of the coupling for $\gamma_{\mathrm{L}}=0$ (thin blue line), $0.5 \omega_{0}$ (solid cyan line), $\omega_{0}$ (dash-dotted green line), $1.5 \omega_{0}$ (dashed magenta line) and $2 \omega_{0}$ (dotted red line), that is the maximal physical value for the model we are considering. We see that going from the undamped to the overdamped regime, the number of virtual photons only diminishes of roughly the $25 \%$. b Trajectory drawn by the eigenfrequencies in the complex plane, for $c k=\omega_{0}$ and $\gamma_{L}=\omega_{0}$, while varying $\omega_{c}$. The black symbols in $\mathbf{a}, \mathbf{b}$ correspond to the same values of $\omega_{\mathrm{c}}$. No visible discontinuity is present in the virtual photon population passing from the weak to the strong coupling regime

Numerical results for Lorentzian resonances. In order to explore the physical content of Eq. (1) we apply it to a medium described by a dissipative Lorentz dielectric function

$$
\epsilon_{\mathrm{L}}(\omega)=1+\frac{\omega_{\mathrm{c}}^{2}}{\omega_{0}^{2}-\omega^{2}-i \gamma_{\mathrm{L}} \omega},
$$

which is a medium containing a single, dispersionless optically active resonance of frequency $\omega_{0}$, coupling strength $\omega_{c}$ and linewidth $\gamma_{\mathrm{L}}$. It is well known that the spectrum of a medium described by Eq. (3) consists of two polaritonic branches, whose real frequencies cross or anticross accordingly to whether the system is in the weak $\left(\gamma_{\mathrm{L}}>2 \omega_{\mathrm{c}}\right)$ or in the strong $\left(\gamma_{\mathrm{L}}>2 \omega_{\mathrm{c}}\right)$ coupling regime, as shown in Fig. 1 . With the appropriate choice of parameters, such a model can be used to describe, at least qualitatively, all linear dielectric condensed matter systems in which strong and ultrastrong coupling have been achieved to date. For historical reasons ${ }^{2}$ the threshold of ultrastrong coupling is usually assumed to be $\omega_{c} \geq 0.2 \omega_{0}$. The poles of the dielectric function in Eq. (3), $\Omega_{0}=\frac{-i \gamma_{\mathrm{L}} \pm \sqrt{4 \omega_{0}^{2}-\gamma_{\mathrm{L}}^{2}}}{2}$, corresponding to the complex frequencies of the lossy matter resonance, have a real component only for $\gamma_{\mathrm{L}}<\gamma_{\max }=2 \omega_{0}$. For $\gamma_{\mathrm{L}}>\gamma_{\max }$ the resonance thus becomes overdamped, the resonant frequency ill defined, and the analytic properties which allow to derive Eq. (1) do not apply anymore. Normally this is a sign that the dissipative
Lorentz model is not adapted to describe the system under investigation and in the following we will thus take $\gamma_{\max }$ as the largest physically meaningful value of the damping.

In Fig. 2a we plot the number of virtual photons $N_{k}$ in the resonant mode $c k=\omega_{0}$ as a function of the light-matter coupling strength $\omega_{c}$. We recognise the expected, initially quadratic dependency over the coupling coefficient $\omega_{c}{ }^{1}$. Different lines relate to different values of $\gamma_{\mathrm{L}}$ ranging from 0 (thin blue line) to $\gamma_{\max }$ (dotted red line). We see that, while losses do have a clear effect upon $N_{k}$, even in the case of an overdamped oscillator the virtual photon population is only diminished by roughly $25 \%$ when compared with the nondissipative case.

The results in the case in which also a photonic linewidth $\gamma_{\mathrm{P}}$ is present, obtained from the general approach developed in the Supplementary Note 4, can be found in the Supplementary Fig. 1. Those results show that also in this more general case our conclusions remain valid. A sizeable virtual photon population in fact remains, reduced at most by $50 \%$ when $\gamma_{L}=\gamma_{P}=\gamma_{\max }$ and the light and matter resonances are both overdamped. Moreover from Supplementary Fig. 1 we can see that in the considered parameter range $N_{k}$ essentially depends on the total linewidth $\gamma_{L}+\gamma_{P}$. The results in Fig. 2a thus generalise to this more general case if one considers the total linewidth instead that the matter one.

As the coupling $\omega_{\mathrm{c}}$ is varied from 0 to $\omega_{0}$ we expect the system, at least for the small and intermediate values of $\gamma_{\mathrm{L}}$, to transition from the weak to the strong coupling regime. Still no 

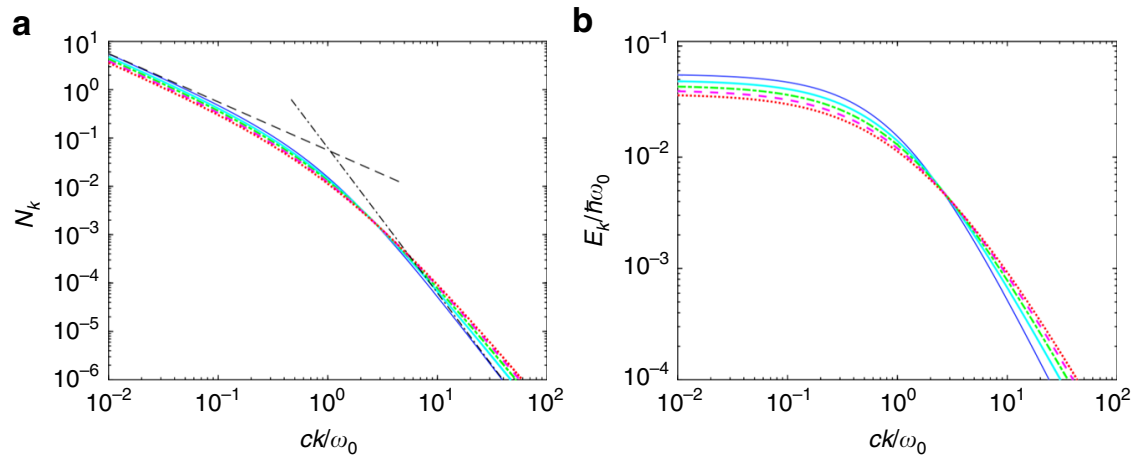

Fig. 3 Virtual photons in the detuned Lorentz model. a Number of virtual photons in the ground state as a function of the photonic wavevector for $\omega_{\mathrm{c}}=$ $0.5 \omega_{0}$ and different values of the losses. The black dashed and dash-dotted lines are the small and large $k$ expansions. $\mathbf{b}$ Photonic energy per mode $E_{k}=\hbar c k N_{k}$ as a function of the photonic wavevector for $\omega_{c}=0.5 \omega_{0}$ and different values of the losses. In both images $\gamma_{L}=0$ (thin blue line), $0.5 \omega_{0}$ (solid cyan line), $\omega_{0}$ (dash-dotted green line), $1.5 \omega_{0}$ (dashed magenta line) and $2 \omega_{0}$ (dotted red line)

discontinuity is observed in $N_{k}$ showing that strong coupling has no direct effect on the virtual photon population. This can be confirmed from Fig. $2 \mathrm{~b}$ where we plot the trajectory of the two complex polaritonic eigenfrequencies in the complex plane for $\gamma_{\mathrm{L}}$ $=\omega_{0}$, as $\omega_{\mathrm{c}}$ is varied, identifying with different symbols specific values marked in Fig. 2a. We can clearly see a transition from the weak to the strong coupling regime as the two eigenfrequencies transition from having different loss rates but similar frequencies to the opposite case.

Numerical results for strongly detuned systems. We verified that losses, even when larger than the light-matter coupling, have only a limited effect on the virtual population of the resonant photonic mode $c k=\omega_{0}$. In order to ascertain if this also remains true out-of-resonance, in Fig. $3 \mathrm{a}$ we plot $N_{k}$ as a function of $\frac{c k}{\omega}$ over four orders of magnitude for a coupling $\omega_{\mathrm{c}}=0.5 \omega_{0}$ and values of the dissipation covering all the range between 0 and $\gamma_{\text {max }}$. We verify again that dissipation does not have any qualitative impact, and also its quantitative effect is negligible for a plot over multiple orders of magnitude of the wavevector. More important, we do not see any sign of resonant enhancement of virtual photon population. This can be understood from the fact that the mixing of the vacuum state with states containing photonic excitations is due to the antiresonant terms of the Hamiltonian, and thus no resonance condition should be expected. Performing a perturbative development in inverse powers of $k$ (now justified as we are interested in extremal values of $k$ ) from of the dissipationless version of Eq. (1) we can find the asymptotic behaviours

$$
N_{k \rightarrow 0}=\frac{\omega_{\mathrm{c}}^{2}}{4 c k \sqrt{\omega_{0}^{2}+\omega_{\mathrm{c}}^{2}}}, \quad N_{k \rightarrow \infty}=\frac{\omega_{0} \omega_{\mathrm{c}}^{2}}{4 c^{3} k^{3}},
$$

plotted as black lines in Fig. 3a. Those results are consistent with the perturbative calculation in the dispersionless case ${ }^{58}$, predicting a larger squeezing for red-detuned resonators. They offer a first proof to a very recent conjecture by Roberto Merlin, linking the dynamical Casimir effect to the problem of orthogonality catastrophes, and predicting the presence of an infrared divergence in the number of generated low-energy photons ${ }^{64}$. In Fig. $3 \mathrm{~b}$ we plot instead $E_{k}=\hbar c k N_{k}$, that is the photonic energy per mode, showing that it is also a monotonously decreasing function of the photonic wavevector $k$, saturating at $E_{\max }=$ $\frac{\hbar \omega_{c}^{2}}{4 \sqrt{\omega_{0}^{2}+\omega_{c}^{2}}}$ for very red-detuned photons.

In summary, we demonstrated that the population of virtual photons present in the ground state of an ultrastrongly coupled system is solid against dissipation. Those results show that ultrastrong-coupling physics can be observed in losses-dominated systems, in which strong coupling is not achievable.

\section{Methods}

Calculation of virtual photon population. In order to derive the formula in Eq. (1) we can start by Huttner and Barnett's diagonalisation method ${ }^{65}$, which extended Hopfield's approach ${ }^{66}$ to the case of a dispersive-dissipative dielectric. For sake of clarity we consider an homogeneous isotropic dielectric medium, although the extension to the inhomogeneous case does not present any fundamental issue ${ }^{67}$. The derivation, detailed in the Supplementary Note 1, starts from an Hamiltonian describing the electromagnetic field coupled to an optically active transition. The latter is coupled to a reservoir of harmonic oscillators which act as a bath in which energy can be dissipated. Introducing annihilation operators $\hat{C}(\mathbf{k}, \omega)$ for excitations of wavevector $\mathbf{k}$ and frequency $\omega$, obeying bosonic commutation relations

$$
\left[\hat{C}(\mathbf{k}, \omega), \hat{C}^{\dagger}\left(\mathbf{k}^{\prime}, \omega^{\prime}\right)\right]=\delta\left(\mathbf{k}-\mathbf{k}^{\prime}\right) \delta\left(\omega-\omega^{\prime}\right),
$$

and using a method originally due to Fano ${ }^{68}$, such an Hamiltonian can be put in the diagonal form

$$
\hat{H}=\sum_{\mathbf{k}} \int_{0}^{\infty} \mathrm{d} \omega \hbar \omega \hat{C}^{\dagger}(\mathbf{k}, \omega) \hat{C}(\mathbf{k}, \omega) .
$$

The linear transformation used to diagonalise the system can then be inverted, allowing us to express the photonic operators as linear combinations of the $\hat{C}(\mathbf{k}, \omega)$ as

$$
\hat{a}(\mathbf{k})=\int_{0}^{\infty} \mathrm{d} \omega\left[\tilde{\alpha}_{0, k}^{*}(\omega) \hat{C}(\mathbf{k}, \omega)-\tilde{\beta}_{0, k}(\omega) \hat{C}^{\dagger}(-\mathbf{k}, \omega)\right],
$$

with

$$
\begin{aligned}
& \tilde{\alpha}_{0, k}(\omega)=\sqrt{\frac{\omega_{c}^{2}}{c k}}\left(\frac{\omega+c k}{2}\right) \frac{\zeta(\omega)}{\epsilon^{*}(\omega) \omega^{2}-c^{2} k^{2}}, \\
& \tilde{\beta}_{0, k}(\omega)=\sqrt{\frac{\omega_{c}^{2}}{c k}}\left(\frac{\omega-c k}{2}\right) \frac{\zeta(\omega)}{\epsilon^{*}(\omega) \omega^{2}-c^{2} k^{2}},
\end{aligned}
$$

where the complex dielectric function is

$$
\epsilon(\omega)=1+\frac{\omega_{\mathrm{c}}^{2}}{2 \omega} \int_{-\infty}^{\infty} \mathrm{d} \omega^{\prime} \frac{\left|\zeta\left(\omega^{\prime}\right)\right|^{2}}{\omega^{\prime}\left(\omega^{\prime}-\omega-i 0^{+}\right)},
$$

and the functional form of $\zeta(\omega)$ can be found in the Supplementary Note 1 . Exploiting the definition of ground state $\hat{C}(\mathbf{k}, \omega)|G\rangle=0$, we can calculate the number of virtual photons with wavevector $\mathbf{k}$ as

$$
N_{k}=\left\langle G\left|\hat{a}^{\dagger}(\mathbf{k}) \hat{a}(\mathbf{k})\right| G\right\rangle=\int_{0}^{\infty} \mathrm{d} \omega\left|\tilde{\beta}_{0, k}(\omega)\right|^{2}=\int_{0}^{\infty} \mathrm{d} \omega \frac{(\omega-k c)^{2}}{2 \pi k c} \frac{\operatorname{Im}[\epsilon(\omega)] \omega^{2}}{\left|\epsilon(\omega) \omega^{2}-c^{2} k^{2}\right|^{2}},
$$


where Im denotes the imaginary part, and we use Eq. (9) and the Sokhotski-Plemelj theorem to write

$$
\operatorname{Im}[\epsilon(\omega)]=\frac{\omega_{\mathrm{c}}^{2} \pi|\zeta(\omega)|^{2}}{2 \omega^{2}} .
$$

Comparing Eq. (7) to Eq. (10) we can verify that the ground state virtual photon population is the square of the mixing coefficient between annihilation and creation operators. The quantity in Eq. (10) is thus a general measure of the hybridisation between states with different numbers of excitations, which is the key ingredient of most ultrastrong-coupling phenomenology $y^{25-27}$.

As detailed below, the expression in Eq. (10) can be calculated through an integral in the complex plane leading to the result in Eq. (1). Note that the parameter $\omega_{\mathcal{c}}$, which quantifies the light-matter coupling, can be arbitrarily large. Our approach in fact takes into account the diamagnetic $A^{2}$ term, forbidding the onset of superradiant phase transitions in polarisable media ${ }^{69,70}$. In order to verify our results, in the Supplementary Note 2 we compare the dissipationless limit of Eq. (1) to the formula obtained using the original Hopfield theory valid for nondissipative systems, showing that the two results coincide. Moreover in the Supplementary Note 3 we explicitly prove that Eq. (3) is in the form of the dielectric function in Eq. (9), and we can thus consistently apply it to Eq. (1).

Calculation of the integral in the complex plane. The total number of photons in the mode $\mathbf{k}$ from Eq. (10) can be calculated by noticing that the dielectric function calculated at a complex frequency $\Omega$ satisfies the relation ${ }^{65}$

$$
\epsilon(\Omega)=\epsilon^{*}\left(-\Omega^{*}\right),
$$

and thus if $\Omega_{j}$ is a solution of Eq. (2) so are $-\Omega_{j}, \Omega_{j}^{*}$ and $-\Omega_{j}^{*}$. Integrating over a keyhole contour in the complex plane, and developing the burdensome algebra paying attention to chose the principal values of $\Omega_{j}$ to have the brach cut on the positive real axis, we arrive at

$$
\begin{aligned}
N_{k} & =\int_{0}^{\infty} \mathrm{d} \omega \frac{(\omega-\mathrm{ck})^{2}}{2 \pi \mathrm{ck}} \frac{\operatorname{Im}[\epsilon(\omega)] \omega^{2}}{\left|\epsilon(\omega) \omega^{2}-c^{2} k^{2}\right|^{2}} \\
& =\sum_{j}\left\{\operatorname{Im}\left[\frac{\Omega_{j}^{2}-c^{2} k^{2}}{4 \pi c^{3} k^{2}} \frac{\mathrm{d} \Omega_{j}}{\mathrm{dk}}\left(i \pi-2 \log \left(\Omega_{j}\right)\right)\right]-\operatorname{Re}\left[\frac{\Omega_{j}}{2 c^{2} k} \frac{\mathrm{d} \Omega_{j}}{\mathrm{dk}}\right]\right\},
\end{aligned}
$$

where the sum is only over the solutions in the first quadrant. Using the sum rule ${ }^{65}$

$$
\sum_{j} \operatorname{Re}\left[\frac{\Omega_{\mathrm{j}}}{k} \frac{\mathrm{d} \Omega_{\mathrm{j}}}{\mathrm{dk}}\right]=c^{2},
$$

we then arrive to the final result in Eq. (1).

Data availability. The data that support the findings of this study are available from the author upon request.

Received: 28 February 2017 Accepted: 22 September 2017

Published online: 13 November 2017

\section{References}

1. Ciuti, C., Bastard, G. \& Carusotto, I. Quantum vacuum properties of the intersubband cavity polariton field. Phys. Rev. B 72, 115303 (2005).

2. Anappara, A. A. et al. Signatures of the ultrastrong light-matter coupling regime. Phys. Rev. B 79, 201303 (2009).

3. Niemczyk, T. et al. Circuit quantum electrodynamics in the ultrastrongcoupling regime. Nat. Phys. 6, 772-776 (2010).

4. Baust, A. et al. Ultrastrong coupling in two-resonator circuit QED. Phys. Rev. B 93, 214501 (2016)

5. Muravev, V. M., Andreev, I. V., Kukushkin, I. V., Schmult, S. \& Dietsche, W. Observation of hybrid plasmon-photon modes in microwave transmission of coplanar microresonators. Phys. Rev. B 83, 075309 (2011).

6. Scalari, G. et al. Ultrastrong coupling of the cyclotron transition of a $2 \mathrm{D}$ electron gas in a THz metamaterial. Science 335, 1323-1326 (2012).

7. Geiser, M. et al. Ultrastrong coupling regime and plasmon polaritons in parabolic semiconductor quantum wells. Phys. Rev. Lett. 108, 106402 (2012).

8. Schwartz, T., Hutchison, J. A., Genet, C. \& Ebbesen, T. W. Reversible switching of ultrastrong light-molecule coupling. Phys. Rev. Lett. 106, 196405 (2011).

9. Porer, M. et al. Nonadiabatic switching of a photonic band structure: ultrastrong light-matter coupling and slow-down of light. Phys. Rev. B 85, $081302(2012)$
10. Askenazi, B. et al. Ultra-strong lightmatter coupling for designer reststrahlen band. New. J. Phys. 16, 043029 (2014).

11. Gubbin, C. R., Maier, S. A. \& Kéna-Cohen, S. Low-voltage polariton electroluminescence from an ultrastrongly coupled organic light-emitting diode. App. Phys. Lett. 104, 233302 (2014).

12. Gambino, S. et al. Exploring light matter interaction phenomena under ultrastrong coupling regime. ACS Photonics 1, 1042-1048 (2014).

13. Maissen, C. et al. Ultrastrong coupling in the near field of complementary splitring resonators. Phys. Rev. B 90, 205309 (2014).

14. Zhang, X., Zou, C.-L., Jiang, L. \& Tang, H. X. Strongly coupled magnons and cavity microwave photons. Phys. Rev. Lett. 113, 156401 (2014).

15. Goryachev, M. et al. High-cooperativity cavity QED with magnons at microwave frequencies. Phys. Rev. Appl. 2, 054002 (2014).

16. Hagenmüller, D. \& Ciuti, C. Cavity QED of the graphene cyclotron transition. Phys. Rev. Lett. 109, 267403 (2012).

17. De Liberato, S. Perspectives for gapped bilayer graphene polaritonics. Phys. Rev B 92, 125433 (2015).

18. Lambert, N., Emary, C. \& Brandes, T. Entanglement and the phase transition in single-mode superradiance. Phys. Rev. Lett. 92, 073602 (2004).

19. Ashhab, S. Superradiance transition in a system with a single qubit and a single oscillator. Phys. Rev. A 87, 013826 (2013).

20. Vukics, A., Grießer, T. \& Domokos, P. Elimination of the -square problem from cavity QED. Phys. Rev. Lett. 112, 073601 (2014).

21. Feist, J. \& Garcia-Vidal, F. J. Extraordinary exciton conductance induced by strong coupling. Phys. Rev. Lett. 114, 196402 (2015).

22. Orgiu, E. et al. Conductivity in organic semiconductors hybridized with the vacuum field. Nature 14, 1123-1129 (2015).

23. De Liberato, S. Light-matter decoupling in the deep strong coupling regime: The breakdown of the purcell effect. Phys. Rev. Lett. 112, 016401 (2014).

24. García-Ripoll, J. J., Peropadre, B. \& De Liberato, S. Light-matter decoupling and $A^{2}$ term detection in superconducting circuits. Sci. Rep. 5, 125433 (2015).

25. Bamba, M. \& Ogawa, T. Laser under ultrastrong electromagnetic interaction with matter. Phys. Rev. A 93, 033811 (2016).

26. Kockum, A. F., Miranowicz, A., Macrí, V., Savasta, S. \& Nori, F. Deterministic quantum nonlinear optics with single atoms and virtual photons. Phys. Rev. A 95, 063849 (2017).

27. Garziano, L., Ridolfo, A., De Liberato, S. \& Savasta, S. Cavity QED in the ultrastrong coupling regime: photon bunching from the emission of individual dressed qubits. ACS Photonics 4, 2345-2351 (2017).

28. Hutchison, J. A., Schwartz, T., Genet, C., Devaux, E. \& Ebbesen, T. W. Modifying chemical landscapes by coupling to vacuum fields. Angew. Chem. 124, 1592-1596 (2012)

29. Hutchison, J. A. et al. Tuning the work-function via strong coupling. $A d v$. Mater. 25, 2481-2485 (2013).

30. Galego, J., Garcia-Vidal, F. J. \& Feist, J. Cavity-induced modifications of molecular structure in the strong coupling regime. Phys. Rev. X 5, 041022 (2015).

31. Cwik, J. A., Kirton, P., De Liberato, S. \& Keeling, J. Self-consistent molecular adaptation induced by strong coupling. Phys. Rev. A. 93, 033840 (2016).

32. Cortese, E., Lagoudakis, P. G. \& De Liberato, S. Collective optomechanical effects in cavity quantum electrodynamics. Phys. Rev. Lett. 119, 043604 (2017).

33. Yoshihara, F. et al. Superconducting qubit-oscillator circuit beyond the ultrastrong-coupling regime. Nat. Phys. 13, 44-47 (2017).

34. Cirio, M., De Liberato, S., Lambert, N. \& Nori, F. Ground state electroluminescence. Phys. Rev. Lett. 116, 113601 (2016).

35. Hagenmüller, D. All-optical dynamical casimir effect in a three-dimensional terahertz photonic band gap. Phys. Rev. B 93, 235309 (2016).

36. Dodonov, V. V. \& Dodonov, A. V. QED effects in a cavity with a timedependent thin semiconductor slab excited by laser pulses. J. Phys. B 39, 749-766 (2006).

37. De Liberato, S., Ciuti, C. \& Carusotto, I. Quantum vacuum radiation spectra from a semiconductor microcavity with a Time-modulated vacuum rabi frequency. Phys. Rev. Lett. 98, 103602 (2007).

38. De Liberato, S., Gerace, D., Carusotto, I. \& Ciuti, C. Extracavity quantum vacuum radiation from a single qubit. Phys. Rev. A. 80, 053810 (2009).

39. Agnesi, A. et al. MIR: an experiment for the measurement of the dynamical Casimir effect. J. Phys. Conf. 161, 012028 (2009).

40. Faccio, D. \& Carusotto, I. Dynamical Casimir effect in optically modulated cavities. Europ. Phys. Lett. 96, 24006 (2011).

41. Carusotto, I., De Liberato, S., Gerace, D. \& Ciuti, C. Back-reaction effects of quantum vacuum in cavity quantum electrodynamics. Phys. Rev. A 85, 023805 (2012).

42. Auer, A. \& Burkard, G. Entangled photons from the polariton vacuum in a switchable optical cavity. Phys. Rev. B 85, 235140 (2012).

43. Fedortchenko, S. et al. Output squeezed radiation from dispersive ultrastrong light-matter coupling. Phys. Rev. A 94, 013821 (2016). 
44. Stassi, R., Ridolfo, A., Di Stefano, O., Hartmann, M. J. \& Savasta, S. Spontaneous conversion from virtual to real photons in the ultrastrongcoupling regime. Phys. Rev. Lett. 110, 243601 (2013).

45. Garziano, L., Ridolfo, A., Stassi, R., Di Stefano, O. \& Savasta, S. Switching on and off of ultrastrong light-matter interaction: Photon statistics of quantum vacuum radiation. Phys. Rev. A 88, 063829 (2013).

46. Lambrecht, A., Jaekel, M.-T. \& Reynaud, S. Motion induced radiation from a vibrating cavity. Phys. Rev. Lett. 77, 615-618 (1996).

47. Johansson, J. R., Johansson, G., Wilson, C. M. \& Nori, F. Dynamical casimir effect in a superconducting coplanar waveguide. Phys. Rev. Lett. 103, 147003 (2009).

48. Nation, P. D., Johansson, J. R., Blencowe, M. P. \& Nori, F. Stimulating uncertainty: Amplifying the quantum vacuum with superconducting circuits. Rev. Mod. Phys. 84, 1-24 (2012).

49. Felicetti, S. et al. Dynamical Casimir effect entangles artificial atoms. Phys. Rev. Lett. 113, 093602 (2014)

50. Stassi, R., De Liberato, S., Garziano, L., Spagnolo, B. \& Savasta, S. Quantum control and long-range quantum correlations in dynamical Casimir arrays. Phys. Rev. A 92, 013830 (2015).

51. Finazzi, S. \& Carusotto, I. Quantum vacuum emission in a nonlinear optical medium illuminated by a strong laser pulse. Phys. Rev. A 87, 023803 (2013)

52. Jacquet, M. \& König, F. Quantum vacuum emission from a refractive-index front. Phys. Rev. A. 92, 023851 (2015).

53. Steinhauser, J. Observation of quantum Hawking radiation and its entanglement in an analogue black hole. Nat. Phys. 12, 959-965 (2016).

54. Günter, G. et al. Sub-cycle switch-on of ultrastrong light-matter interaction. Nature 458, 178-181 (2009).

55. Peropadre, B., Forn-Diaz, P., Solano, E. \& Garcia-Ripoll, J. J. Switchable ultrastrong coupling in circuit QED. Phys. Rev. Lett. 105, 023601 (2010).

56. Wilson, C. M. et al. Observation of the dynamical Casimir effect in a superconducting circuit. Nature 479, 376-379 (2011).

57. Scalari, G. et al. Superconducting complementary metasurfaces for $\mathrm{THz}$ ultrastrong light-matter coupling. New J. Phys. 16, 033005 (2014).

58. Beaudoin, F., Gambetta, J. M. \& Blais, A. Dissipation and ultrastrong coupling in circuit QED. Phys. Rev. A 84, 043832 (2011).

59. Mamba, M. \& Ogawa, T. System-environment coupling derived by Maxwell's boundary conditions from the weak to the ultrastrong light-matter-coupling regime. Phys. Rev. A 88, 013814 (2013).

60. Mamba, M. \& Ogawa, T. Recipe for the Hamiltonian of system-environment coupling applicable to the ultrastrong-light-matter-interaction regime. Phys. Rev. A 89, 023817 (2014).

61. Mi, X., Cady, J., Zajac, D. M., Deelman, P. W. \& Petta, J. R. Strong coupling of a single electron in silicon to a microwave photon. Science 355, 156-158 (2017).

62. Toida, H., Nakajima, T. \& Komiyama, S. Vacuum rabi splitting in a semiconductor circuit QED system. Phys. Rev. Lett. 110, 066802 (2013).

63. Wallraff, A., Stockklauser, A., Ihn, T., Petta, J. R. \& Blais, A. Comment on vacuum rabi splitting in a semiconductor circuit QED system. Phys. Rev. Lett. 111, 249701 (2013).

64. Merlin, R. Orthogonality catastrophes in quantum electrodynamics. Phys. Rev. A 95, 023802 (2017).
65. Huttner, B. \& Barnett, S. M. Quantization of the electromagnetic field in dielectrics. Phys. Rev. A 46, 4306-4322 (1992).

66. Hopfield, J. J. Theory of the contribution of excitons to the complex dielectric constant of crystals. Phys. Rev. 112, 1555-1567 (1958).

67. Gubbin, C. R., Maier, S. A. \& De Liberato, S. Real-space Hopfield diagonalization of inhomogeneous dispersive media. Phys. Rev. B 94, 205301 (2016).

68. Fano, U. Effects of configuration interaction on intensities and phase shifts. Phys. Rev. 124, 1866-1878 (1956).

69. Rzazewski, K., Wodkiewicz, K. \& Zacowicz, W. Phase transitions, two-level atoms, and the $A^{2}$ term. Phys. Rev. Lett. 35, 432-434 (1975).

70. Bamba, M. \& Ogawa, T. Stability of polarizable materials against superradiant phase transition. Phys. Rev. A 90, 063825 (2014).

\section{Acknowledgements}

I thank Kristín Arnardóttir, Motoaki Bamba, Neill Lambert, Ahsan Nazir and Giacomo Scalari for useful discussions and feedback. I am a University Research Fellow of the Royal Society and I acknowledge the support received from EPSRC grant EP/M003183/1

\section{Author contributions}

S.de L. designed the research, performed the research and wrote the paper.

\section{Additional information}

Supplementary Information accompanies this paper at doi:10.1038/s41467-017-01504-5.

Competing interests: The author declares no competing financial interests.

Reprints and permission information is available online at http://npg.nature.com/ reprintsandpermissions/

Publisher's note: Springer Nature remains neutral with regard to jurisdictional claims in published maps and institutional affiliations.

Open Access This article is licensed under a Creative Commons Attribution 4.0 International License, which permits use, sharing, adaptation, distribution and reproduction in any medium or format, as long as you give appropriate credit to the original author(s) and the source, provide a link to the Creative Commons license, and indicate if changes were made. The images or other third party material in this article are included in the article's Creative Commons license, unless indicated otherwise in a credit line to the material. If material is not included in the article's Creative Commons license and your intended use is not permitted by statutory regulation or exceeds the permitted use, you will need to obtain permission directly from the copyright holder. To view a copy of this license, visit http://creativecommons.org/ licenses/by/4.0/

(C) The Author(s) 2017 